\title{
Laser induced fluorescence spectroscopy of $\mathbf{N}_{2}\left(A^{3} \Sigma_{u}^{+}\right)$ and absolute density calibration by Rayleigh scattering in capacitively coupled rf discharges
}

\author{
S. Nemschokmichal, F. Bernhardt, B. Krames, and J. \\ Meichsner \\ University of Greifswald, Institute of Physics Felix-Hausdorff-Stras̈e 617489 \\ Greifswald \\ E-mail: nemschok@physik.uni-greifswald.de
}

\begin{abstract}
Laser induced fluorescence spectroscopy (LIF) was applied to measure absolute densities of the first metastable $\mathrm{N}_{2}\left(A^{3} \Sigma_{u}^{+}, v=0\right)$ state in capacitively coupled rf discharges at $13.56 \mathrm{MHz}$. The metastable state was transferred to the $B^{3} \Pi_{g}, v=3$ state with a dye laser at $687.44 \mathrm{~nm}$ and the fluorescence of the transition to the $A^{3} \Sigma_{u}^{+}, v=1$ state was observed at $762 \mathrm{~nm}$. The time-depending LIF signal was investigated and the effective lifetime of the $B^{3} \Pi_{g}, v=3$ state was determined by eliminating the delay due to the photomultiplier circuit. The absolute density calibration was done by comparison with RAYLEIGH scattering at the laser wavelength of $687 \mathrm{~nm}$. Therewith, axial density profiles were measured and their dependence on pressure and power was investigated. The metastable densities vary between $10^{12}$ to $10^{13} \mathrm{~cm}^{-3}$ for pressures from 40 to $1000 \mathrm{~Pa}$. Furthermore, small amounts of oxygen ( 0.5 to $2 \%$ ) were added and the influence on the metastable density is discussed.
\end{abstract}

PACS numbers: $52.25 . \mathrm{Os}, 52.25$.Ya, 52.70.Kz, 52.80.Pi

52.25. Os Emission, absorption, and scattering of electromagnetic radiation

52.25.Ya Neutrals in plasmas

52.70.Kz Optical (ultraviolet, visible, infrared) measurements

52.80.Pi High-frequency and RF discharges

Submitted to: J. Phys. D: Appl. Phys. 


\section{Introduction}

Neutral metastable excited atoms and molecules are important carriers of internal energy in a plasma. Due to their large lifetime, they can transfer a large amount of energy from the plasma to surfaces or keep this energy in the afterglow plasma. Especially for larger pressure, they gain importance because of the possibility for exoemission of seed electrons from surfaces, which are important for the ignition of atmospheric pressure discharges. Therefore, it is necessary to quantify metastable densities for the understanding of discharge development.

Taking into consideration the electronic states of molecular nitrogen, the first electronic excited state $A^{3} \Sigma_{u}^{+}$is metastable with a radiative lifetime of seconds and an energy of $6.2 \mathrm{eV}$ with respect to the ground state $X^{1} \Sigma_{g}^{+}$. Just above $(1.2 \mathrm{eV})$, two other triplet states $\left(B^{3} \Pi_{g}, W^{3} \Delta_{u}\right)$ exist[1], but only the $B^{3} \Pi_{g}$ state has visible optical transitions to the $A^{3} \Sigma_{u}^{+}$state (1st positive system)[2] which are accessible for laser induced fluorescence spectroscopy (LIF).

Examples for the LIF application to low pressure nitrogen discharges can be found in $[3,4,5,6]$. They differ in particular in the absolute density calibration, which can be done straight forward by calculation[4] or by a comparison with scattered RAYLEIGH light[5]. Other methods than LIF are recent measurements with threshold ionization mass spectrometry[7], cavity enhanced absorption spectroscopy[8] or cavity ring down spectroscopy $[9,10]$. In particular, for atmospheric pressure discharges the LIF technique is still the most suitable one. Therefore, the simple one laser LIF spectroscopy has been improved to the so called optical-optical double resonance LIF spectroscopy, which makes use of both the first and second positive system[11]. It was applied successfully to a dielectric barrier discharge[12], where the absolute density calibration has been done by a comparison of the emission of the 2nd positive system, caused by the pooling reaction of two $A^{3} \Sigma_{u}^{+}$molecules, with the $\mathrm{NO}-\gamma$ system, caused by collisions of $A^{3} \Sigma_{u}^{+}$ with the NO ground state. This calibrated diagnostics has also been applied to a surface dielectric barrier discharge $[13,14]$. Another possibility in atmospheric pressure discharges is the calibration directly by the pooling reaction, which has been done in a pulsed corona discharge[15, 16].

The main goal of this work is the application of the LIF method together with the calibration by RAYLEIGH scattering in capacitively coupled radio-frequency discharges in nitrogen at pressures up to $1000 \mathrm{~Pa}$ which is above the typical low pressure range. Thereby, the most important challenge is the handling of the large quenching in comparison to the low optical emission. This is especially necessary for a further increase of the pressure up to atmospheric pressure discharges, which should be investigated afterwards.

The paper starts with the detailed description of the experiment which is designed

to analyse the time response of the LIF signal. This analysis gives the optical emission to the ratio of the quenching by the comparison with the scattered laser light. Afterwards, the calibration by RAYLEIGH scattering is done. In the end, the measured axial density 

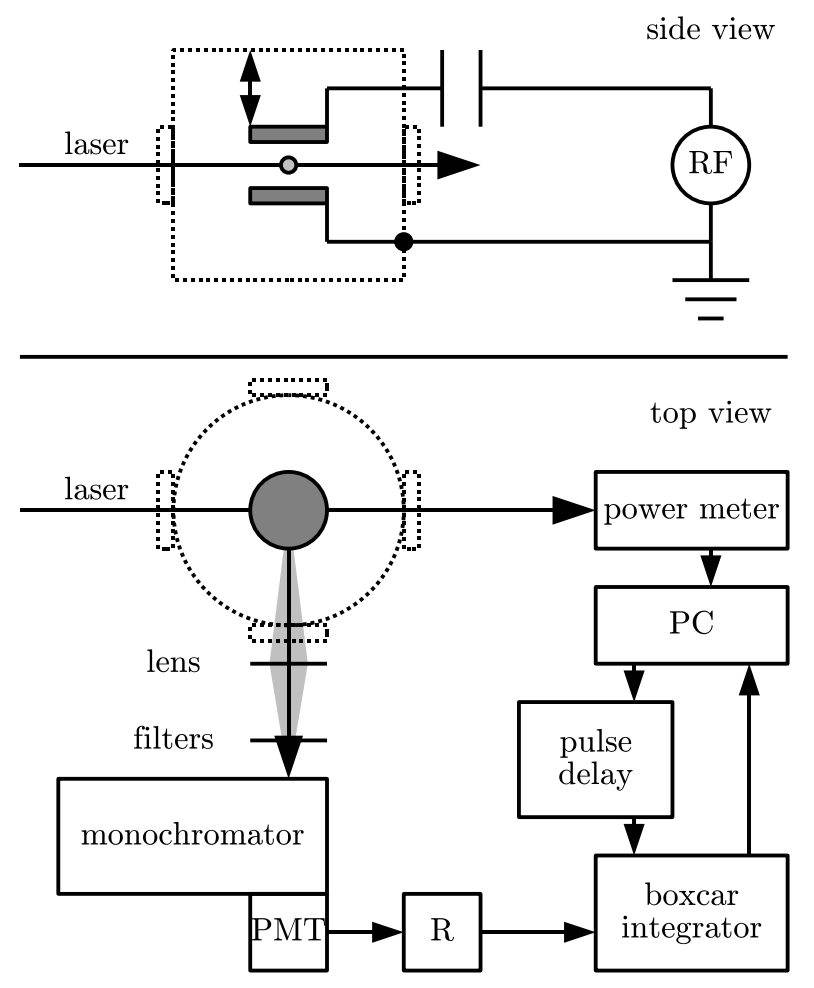

Figure 1. Experimental setup of the discharge and the LIF measurement.

profiles in the capacitively coupled radio-frequency discharge are shown and discussed for different plasma processing parameters. Furthermore, the influence of small oxygen admixtures is discussed, which is known as a strong quencher of the metastable nitrogen state.

\section{Experimental setup}

A sketch of the experimental setup is shown in figure 1, which can be divided into the setup for the capacitively coupled radio frequency (cc-rf) discharge and the laser induced fluorescence (LIF) measurement, consisting of a Nd:YAG pumped dye laser and a combination of a monochromator and a photomultiplier as detector.

\section{1. $C C-R F$ discharge}

The cylindrical stainless steel vacuum chamber (300 mm in diameter, $500 \mathrm{~mm}$ height) was evacuated by a turbo pump (Pfeiffer Vacuum, TMU-260, forepump: LABOVAC $\AA$ ) PK 8 D) to a base pressure below $10^{-4} \mathrm{~Pa}$. Afterwards the chamber was filled with pure nitrogen (pureness of $99.999 \%$ ) to pressures from 40 to $1000 \mathrm{~Pa}$ by means of a mass flow controller (MKS PR4000F and MKS Mass-Flo @ Controller). Furthermore, small admixtures of oxygen up to $2 \%$ were performed by a second mass flow controller. To keep a constant nitrogen gas flow rate of $5 \mathrm{sccm}$ for pure $\mathrm{N}_{2}$ plasma and $10 \mathrm{sccm}$ in the case of $\mathrm{O}_{2}$ admixture, the total pressure was independently controlled by a diaphragm 
pressure gauge (MKS Baratron ${ }^{\circledR}$ Pressure Controller), a butterfly valve (MKS) and a process pump (TRIVAC D25BCSPFPE).

In the centre of the chamber, the discharge was ignited between two parallel plate stainless steel electrodes with diameter of $90 \mathrm{~mm}$ and distance of $10 \mathrm{~mm}$. The lower electrode was powered via a matching network (ENI MW-10D) with a radio-frequency generator (ENI ACG-6B) at a fixed frequency of $13.56 \mathrm{MHz}$. The upper electrode was grounded as well as the walls of the vacuum chamber, wherefore the effective area of the grounded electrode was larger then the powered electrode and an asymmetric capacitively coupled discharge arised. Depending on the applied rf power of between 10 and $80 \mathrm{~W}$ as well as the total pressure the resulting self-bias voltage at the powered electrode was in the range from -15 to $-350 \mathrm{~V}$.

\subsection{Laser induced fluorescence}

The laser system for the excitation consists of a frequency doubled Nd:YAG laser (Quanta Ray GCR 130) with a maximal pulse energy of $170 \mathrm{~mJ}$ as pump laser and a tunable dye laser (LAS LDL 20505). The used dye was pyridine 1 which allowed the tuning of the laser wavelength of between 670 and $730 \mathrm{~nm}$ with a linewidth of about $0.6 \mathrm{~cm}^{-1}$, beam diameter of $1.5 \mathrm{~mm}$ and pulse energy up to $30 \mathrm{~mJ}$. In the experiment a maximum laser energy of $10 \mathrm{~mJ}$ was used to avoid stimulated emission and full saturation of the LIF signal.

The laser beam was guided parallel through the electrodes, crossing the discharge axis. To measure the density of the state, the laser wavelength was tuned to $687.44 \mathrm{~nm}$, where the band head of the $A^{3} \Sigma_{u}^{+}, v=0 \rightarrow B^{3} \Pi_{g}, v=3$ ( $\mathrm{Q}_{12}$ and $\mathrm{P}_{11}$ branches) is located. Perpendicular to the laser beam, the fluorescence was focused by means of a lens (focal length $150 \mathrm{~mm}$ ) to the entrance slit of a $500 \mathrm{~mm}$ monochromator (ARC Acton Research Corporation, SpectraPro ${ }^{\circledR}$, grating $1200 \mathrm{~mm}^{-1}$, bandwidth $5 \mathrm{~nm}$ ) and detected via a photomultiplier (PMT, Hamamatsu R928) afterwards.

The monochromator was tuned to a wavelength of $762 \mathrm{~nm}$, which corresponds to the band head of the $B^{3} \Pi_{g}, v=3$ to $A^{3} \Sigma_{u}^{+}, v=1$ transition. In addition, an RG9 filter was placed in front of the spectrometer to cut off the scattered laser light, which partially transmits the spectrometer even at the wavelength of $762 \mathrm{~nm}$.

For the calibration procedure, the plasma was switched off and the spectrometer was set to $687.4 \mathrm{~nm}$ and the RG9 filter was removed to measure the intensity of the scattered laser light by pure nitrogen at room temperature. Because of the large intensity of the scattered light, a neutral density filter (NG3) was build in to avoid saturation effects in the PMT. Additionally, it should be mentioned here, that the linear laser polarization was perpendicular to the observation direction to get a sufficient large RAYLEIGH scattering intensity, considering the anisotropy of the differential scattering cross section.

The output of the PMT was connected via a tunable resistance $R \approx 1 \mathrm{k} \Omega$ with the ground, and the potential drop across the resistance was input to a boxcar integrator 


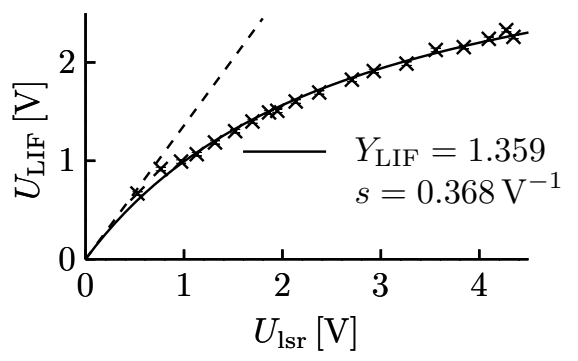

Figure 2. Saturation of the LIF signal depending on laser energy. The fit gives the saturation parameter $s$.

(SRS SR 250, input impedance: $1 \mathrm{M} \Omega$ ). The gate delay of the boxcar integrator with respect to the laser pulse was varied via a pulse delay generator (SRS, DG535) to measure the time dependent LIF signal respectively the time dependent scattered signal for comparison. The voltage output of the boxcar integrator was measured with an A-D converter card (Keithley DAS-801) inside a PC.

\section{Results and discussion}

In this part the separated steps for the determination of absolute densities are discussed, starting with the consideration of the saturation effect, the analysis of the timedepending LIF signal, the comparison of the optics and the cross sections as well as the RAYLEIGH measurement itself. Afterwards, axial metastable density profiles for different plasma processing parameters are presented and interpreted in comparison with plasma induced optical emission. At last, the influence of oxygen admixtures on the absolute metastable density is discussed.

\subsection{Saturation effect}

In a first approach, the LIF signal is proportional to the laser pulse energy. This is true for small laser energies, but for larger ones the strong laser field causes also induced emission and therefore a smaller density of the excited state. The result is a saturation curve as shown in figure 2, where the measured LIF voltage $U_{\text {LIF }}$ is plotted versus the voltage of the laser energy detector $U_{\mathrm{lsr}}$, which is proportional to the laser pulse energy:

$$
U_{\mathrm{LIF}}=Y_{\mathrm{LIF}} \frac{U_{\mathrm{lsr}}}{1+s U_{\mathrm{lsr}}}
$$

The dimensionless quantity $Y_{\text {LIF }}$ is the LIF signal per laser energy without the saturation effect. The parameter $s$ describes the saturation level. It was calculated once from a fit, so that the LIF signal $Y_{\text {LIF }}$ could be calculated directly from $U_{\text {LIF }}$ for the following measurements. This was especially necessary because of the changing laser intensity and its temporal drift to smaller laser energies due to the heating of the laser after longer operation. 


\subsection{Time response of LIF signal}

The LIF signal depends strongly on the ratio of the fluorescence intensity at the observed wavelength of $762 \mathrm{~nm}$ and the remaining loss processes of the excited $B^{3} \Pi_{g}, v=3$ state, such as the fluorescence at other wavelengths and the collisional quenching. In the case that the collisions of the considered $B^{3} \Pi_{g}, v=3$ state with other species dominate and any additional production after the laser pulse at $t_{0}$ is negligibly, than the rate equation for its density is quite simple:

$$
\frac{\mathrm{d} n_{B_{3}}}{\mathrm{~d} t}=-\frac{1}{\tau_{\text {eff }}} n_{B_{3}} .
$$

The effective lifetime $\tau_{\text {eff }}$ depends on the radiative lifetime $\tau_{\mathrm{r}}$ of $B^{3} \Pi_{g}, v=3$ and the collision rates $k_{q}$ of the excited state with the quenchers $q$ and their densities $n_{q}$ :

$$
\frac{1}{\tau_{\mathrm{eff}}}=\frac{1}{\tau_{\mathrm{r}}}+\sum_{q} k_{q} n_{q} .
$$

In this experiment, the short laser pulse duration $(<10 \mathrm{~ns})$ can be neglected in comparison with the effective lifetime of the $B^{3} \Pi_{g}, v=3$ state. At atmospheric pressure, this assumption is no longer valid and the time dependent laser pulse has to be taken into account in equation (2), too.

The solution of the rate equation is an exponential decay with the effective lifetime $\tau_{\text {eff }}$ after the laser pulse which results also in an exponential decay for the LIF intensity $I_{\mathrm{LIF}}(t)=A_{B_{3} \rightarrow A_{1}} n_{B_{3}}(t)$,

$$
I_{\mathrm{LIF}}(t)=\hat{I}_{\mathrm{LIF}} \mathrm{e}^{-\frac{t-t_{0}}{\tau_{\text {eff }}}}
$$

where $A_{B_{3} \rightarrow A_{1}}=6.446 \cdot 10^{4} \mathrm{~s}^{-1}[17]$ is the EINSTEIN coefficient for spontaneous emission from $B^{3} \Pi_{g}, v=3$ to $A^{3} \Sigma_{u}^{+}, v=1$.

In the experiment, the light passes the spectrometer and releases charges in the PMT, that drive an electric current via the external resistor to the ground. This is a simple $\mathrm{RC}$ circuit with a time constant of $\tau_{\mathrm{PMT}}=R C$, which has been proven by varying the resistor $R$. Therefore, the voltage $u_{\mathrm{LIF}}$ across the resistor has an additional exponential decay due to the electrical circuit of the PMT and the external resistor, which has to be calculated as a convolution of this exponential decay with the LIF intensity:

$$
u_{\mathrm{LIF}}(t) \propto \int_{t_{0}}^{t} I_{\mathrm{LIF}}\left(t^{\prime}\right) \mathrm{e}^{-\frac{t-t^{\prime}}{\tau_{\mathrm{PMT}}}} \mathrm{d} t^{\prime},
$$

Calculation of equation (5) results in a difference of two exponential decays with the time constants $\tau_{\text {eff }}$ due to the lifetime of the excited state and $\tau_{\text {PMT }}$ due to the PMT circuit, which are in the microsecond respectively sub-microsecond time scale:

$$
\begin{aligned}
u_{\text {LIF }}(t) & =\hat{u}_{\text {LIF }} \frac{A_{B_{3} \rightarrow A_{1}}}{\tau_{\text {eff }}^{-1}} \frac{\tau_{\text {eff }}}{\tau_{\text {eff }}-\tau_{\text {PMT }}} \\
& \times\left(\mathrm{e}^{-\frac{t-t_{0}}{\tau_{\text {eff }}}}-\mathrm{e}^{-\frac{t-t_{0}}{\tau_{\mathrm{PMT}}}}\right) .
\end{aligned}
$$




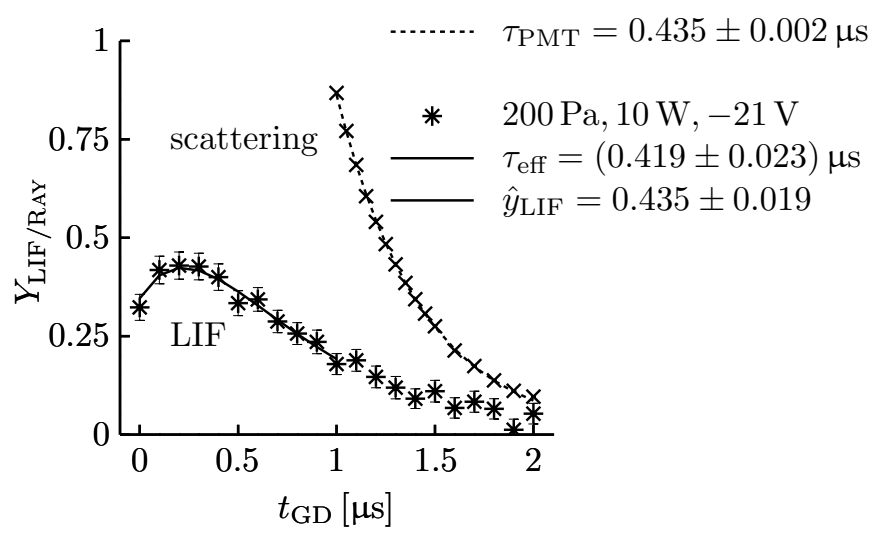

Figure 3. LIF voltage from the boxcar integrator depending on the gate delay of the boxcar for the LIF signal and the signal of the scattered laser light. The lines represent fits of the theoretical time responses.

Here, the ratio of the fluorescence rate, represented by the EINSTEIN coefficient $A_{B_{3} \rightarrow A_{1}}$ to the overall loss rate $\tau_{\text {eff }}^{-1}$, is of importance to consider the losses of $B^{3} \Pi_{g}, v=3$ by collisional quenching.

In the later calibration of the LIF signal by means of the RAYLEIGH scattering, the different temporal behaviour of the scattered light has to be considered. The scattered light appears immediately when the laser pulse takes place, so that there is no delay as for the fluorescence. In addition, the decay time of the PMT circuit is adjusted to be in the microsecond range, so that the laser pulse duration can be neglected again. Therefore, the voltage across the resistor shows a single exponential decay determined by the PMT circuit itself:

$$
u_{\text {scatt }}(t)=\hat{u}_{\text {scatt }} \mathrm{e}^{-\frac{t-t_{0}}{\tau_{\text {PMT }}}}
$$

As mentioned before, the voltages $u_{\text {LIF }}$ and $u_{\text {RAY }}$ across the resistance are not the measured ones, therefore, these voltages have additionally to be integrated, which yield the voltages at the output of the boxcar integrator, with a gate delay $t_{\mathrm{GD}}$ and a gate width $t_{\mathrm{GW}}$ :

$$
U_{\mathrm{LIF} / \text { scatt }}\left(t_{\mathrm{GD}}\right)=\frac{1}{t_{\mathrm{GW}}} \int_{t_{\mathrm{GD}}}^{t_{\mathrm{GD}}+t_{\mathrm{GW}}} u_{\mathrm{LIF} / \mathrm{scatt}}(t) \mathrm{d} t .
$$

The integrated voltage for the scattered signal remains an exponential decay with $\tau_{\text {PMT }}$ as the time constant and another factor in front of the exponential function. The temporal signal for the LIF remains mainly the same in comparison with (6), but it starts with a finite value at $t_{\mathrm{GD}}=0$ because of the finite gate width of the boxcar integrator. This is clearly recognizable in figure 3, where the scattered signal and the LIF signal are plotted versus the gate delay of the boxcar integrator.

In addition, the LEVENBERG-MARQUARDT algorithm is used to plot two fit functions in figure 3 for the LIF and scattering signal from equation (8). The agreement 


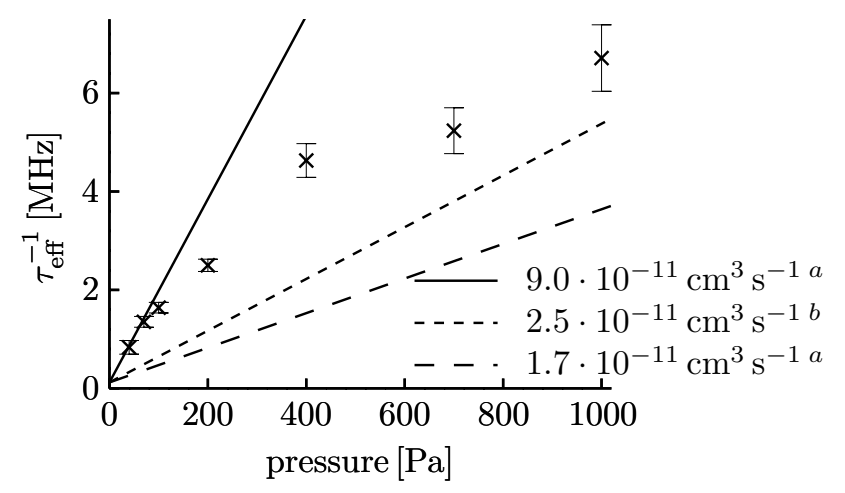

Figure 4. Effective decay rates $\tau_{\text {eff }}^{-1}$ of the excited $B^{3} \Pi_{g}, v=3$ state depending on pressure. The straight lines represent quenching rate coefficients from literature (a [18], b [19]).

of the exponential fit with the values from the measurement of the scattered light is very good and allows to find out the PMT decay time constant $\tau_{\mathrm{PMT}}$. Following, the fit of the LIF signal is done and the effective lifetime $\tau_{\text {eff }}$ and the signal strength $\hat{y}_{\text {LIF }}$ were extracted. There, the signal strength $\hat{y}_{\text {LIF }}$ represents a kind of original signal strength if there would not be any delay due to the lifetime of the excited state and the PMT circuit. For fitting the LIF signal only the measurements until $1 \mu$ s are taken into account to avoid the influence of backward processes, for example from the $W^{3} \Delta_{u}$ state (see below).

Considering equation (3) and assuming that the main quenching partner is the background gas (ground state $X^{1} \Sigma_{g}^{+}$with the density $n_{X}=p / k_{\mathrm{B}} T_{\text {gas }}$ ), then the effective decay rate $\tau_{\text {eff }}^{-1}$ should depend linear on pressure. To proof this, the effective decay rates $\tau_{\text {eff }}^{-1}$ are plotted versus pressure in figure 4.

First of all, one can see that the overall decay rate $\tau_{\text {eff }}^{-1}$ is dominated by quenching and only the small intercept is caused by optical transitions. For example, the overall decay rate at $1000 \mathrm{~Pa}$ of $\tau_{\text {eff }}^{-1} \approx 6.5 \mathrm{MHz}$ is about 100 times larger than the EINSTEIN coefficient for the considered optical transition at $762 \mathrm{~nm}$.

Obviously, the effective decay rates follow not a straight line. For comparison, some curves are drawn using quenching rates from literature, but these rates differ strongly from the measured values. One reason might be the collisional coupling of the $B^{3} \Pi_{g}$ state to the $W^{3} \Delta_{u}$ state, observed already in $[20,21,18]$ :

$$
B^{3} \Pi_{g}+X^{1} \Sigma_{g}^{+} \rightleftharpoons W^{3} \Delta_{u}+X^{1} \Sigma_{g}^{+} .
$$

This could also explain the small increase in figure 3 of the LIF signal after $1 \mu \mathrm{s}$. In [21], the rate coefficient for the backward reaction in (9) is assumed to be much smaller than for the forward reaction, which might result in a larger $B^{3} \Pi_{g}$ density late after the laser pulse.

The change of the effective lifetime has to be considered together with the variation of the plasma processing parameters. As it is obvious and shown, the effective lifetime 
depends on pressure. Furthermore, an effect due to the larger gas temperature at larger rf power is expected. Firstly, the mean thermal velocity of the background gas depends on temperature $\left(v_{\text {th }} \propto \sqrt{T_{\text {gas }}}\right)$, which yields in a first approximation to a temperature dependent quenching rate coefficient of $k_{X}$ proportional to $\sqrt{T_{\text {gas }}}$. In addition, the density of the most important quencher, the background gas in its ground state $X^{1} \Sigma_{g}^{+}$ depends on pressure by the ideal gas law for constant pressure: $n=p / k_{\mathrm{B}} T_{\text {gas }}$. As a result, both effects give an effective decay rate $\tau_{\text {eff }}^{-1} \approx k_{X} n_{X}$ proportional to $T^{-1 / 2}$. The analysis of the rotational temperature from the $B^{3} \Pi_{g}, v=2 \rightarrow A^{3} \Sigma_{u}^{+}, v=0$ and $B^{3} \Pi_{g}, v=3 \rightarrow A^{3} \Sigma_{u}^{+}, v=1$ bands provide a temperature growth of $200 \mathrm{~K}$ for a power increase from 10 to $70 \mathrm{~W}$ at $200 \mathrm{~Pa}$. Unfortunately, the rotational temperature for low rf power $(10 \mathrm{~W})$ could not be determined and the gas temperature of $350 \mathrm{~K}$ slightly above the room temperature was assumed. For this, a factor of $\sqrt{550 / 350} \approx 1.25$ remains from the discussion above. To proof this, the rf power at $200 \mathrm{~Pa}$ was varied, but only a small increase of $\tau_{\text {eff }}$ within the error range was observed. Therefore, the effective lifetime is taken to be constant for selected pressure. Nevertheless, this might be a reason for the deviations from the literature vaules in figure 4 for 700 and $1000 \mathrm{~Pa}$, because at larger pressures also larger rf power was necessary for sustaining the discharge and stronger gas heating is expected.

Another influence on the effective lifetime might be due to the admixtures of oxygen. As it is known, the quenching of $A^{3} \Sigma_{u}^{+}$by oxygen is large, but the quenching of the excited state $B^{3} \Pi_{g}$ by oxygen is in the same order as the quenching by nitrogen. Consequently, the small admixtures of oxygen should not affect the effective lifetime. This was proofed experimentally, and there are no differences of the effective lifetimes with and without oxygen admixture within the error range.

\subsection{Absolute density calibration}

The absolute density calibration is done by the comparison of the LIF signal at $762 \mathrm{~nm}$ to the RAYLEIGH scattering signal at $687.4 \mathrm{~nm}$ to avoid the calculation of the interaction volume between the laser and the detection cone. The whole formula is given by

$$
n_{A_{0}}=\hat{y}_{\mathrm{LIF}} \frac{T_{\mathrm{RAY}} Q_{\mathrm{RAY}}}{T_{\mathrm{LIF}} Q_{\mathrm{LIF}}} \frac{3}{2} \frac{\sigma_{\mathrm{RAY}}}{\sigma_{\mathrm{LIF}}} \frac{n_{\mathrm{RAY}}}{\hat{y}_{\mathrm{RAY}}}
$$

for the density of the $A^{3} \Sigma_{u}^{+}, v=0$ state. First of all, it enters the signal strength $\hat{y}_{\text {LIF }}$, calculated from the integrated boxcar signal by use of the effective lifetime, the PMT decay time and the saturation factor. Furthermore, the ratios of the transmission optics at $687 \mathrm{~nm}\left(T_{\mathrm{RAY}}\right)$ respectively $762 \mathrm{~nm}\left(T_{\mathrm{LIF}}\right)$ and the quantum efficiencies of the $\operatorname{PMT}\left(Q_{\mathrm{RAY}}, Q_{\mathrm{LIF}}\right)$ appear, as well as the ratio of the cross sections $\sigma_{\mathrm{RAY}}$ and $\sigma_{\mathrm{LIF}}$. The additional factor $3 / 2$ will be discussed below. At the end, the ratio of the known density of the RAYLEIGH scatterer $n_{\text {RAY }}$ to the RAYLEIGH signal strength $\hat{y}_{\text {RAY }}$ is included.

The ratio of the optics was determined by the comparison of the signal from a cold light lamp with known temperatures of between 2650 and $3200 \mathrm{~K}$ for the different wavelengths. This yielded a factor of $0.37 \pm 0.02$ for $T_{\mathrm{RAY}} Q_{\mathrm{RAY}} / T_{\mathrm{LIF}} Q_{\mathrm{LIF}}$, because of the 


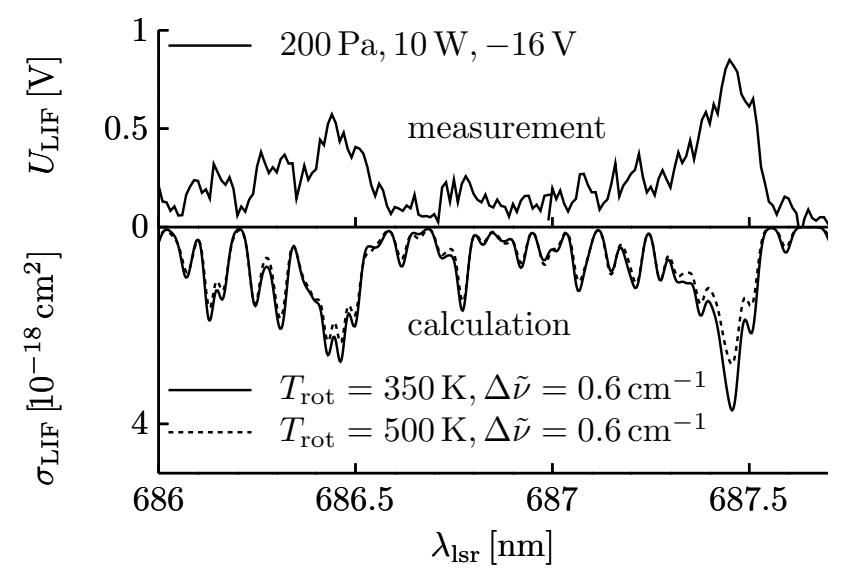

Figure 5. Comparison of the relative LIF intensity with a laser scan and the simulated LIF cross sections.

better quantum efficiency for the lower wavelength, partially compensated by the better transmission of the spectrometer at $762 \mathrm{~nm}$ (grating blazed for $750 \mathrm{~nm}$ ). Additionally, a neutral density filter was used for the RAYLEIGH scattering experiment to avoid saturation inside the PMT.

For the calculation of the LIF cross section $\sigma_{\mathrm{LIF}}$ at the laser wavelength $\lambda_{\mathrm{lsr}}=$ $2 \pi c / \omega_{\text {lsr }}$ the convolution of the laser profile with the line profiles of the $A^{3} \Sigma_{u}^{+}, v=0 \rightarrow$ $B^{3} \Pi_{g}, v=3$ rotational band is essential:

$$
\sigma_{\mathrm{LIF}}\left(\omega_{\mathrm{lsr}}\right)=\sum_{k} F_{k} \int_{\omega} \sigma_{k}(\omega) G\left(\omega, \omega_{\mathrm{lsr}}\right) \mathrm{d} \omega .
$$

The factor $F_{k}$ is the part of the rotation line at $\omega_{k}$ of the whole $A^{3} \Sigma_{u}^{+}, v=0 \rightarrow B^{3} \Pi_{g}, v=$ 3 band, which implies $\sum_{k} F_{k}=1$. These factors $F_{k}$ are given by a numerical calculation which is based on data from high resolution FOURIER spectrometry [22] and was already used for this calibration method[5]. The first factor

$$
\sigma_{k}(\omega)=\frac{\pi e^{2} f_{j i}}{2 \varepsilon_{0} m_{e} c_{0}} f\left(\omega, \omega_{k}\right)
$$

in the convolution integral (11) represents the frequency dependent LIF cross section for a line centered at $\omega_{k}$. It depends on the oscillator strength $f_{j i}=1.404 \cdot 10^{-4}[17]$ and the Doppler broadened line profile $f\left(\omega, \omega_{k}\right)$. As visible in equation (11), the cross section for one line was convoluted with the laser profile $G\left(\omega, \omega_{\text {lsr }}\right)$, which was assumed to be a GAUSSIAN distribution with a FWHM of $\Delta \tilde{\nu} \approx 0.6 \mathrm{~cm}^{-1}$, measured by a laser scan of a optogalvanic calibration lamp with neon.

To proof the calculation, the cross section for two rotational temperatures is plotted together with a measured laser scan in figure 5. The agreement with the simulation is very well, so it is possible to take the cross section from the simulation at the laser wavelength of $687.44 \mathrm{~nm}$. Unfortunately, the cross section decreases with temperature due to the changing distribution of the rotational levels within the vibrational state. 
Therefore, one has to take care about this effect if the power of the discharge increases significantly.

The calculation of the cross section for the RAYLEIGH scattering is less complicated. It can be done as by MiLES et al. [23] by

$$
\sigma_{\mathrm{RAY}}=\frac{8 \pi^{3}}{3 \varepsilon_{0}^{2} \lambda^{4}} \alpha^{2}\left(\frac{6-3 \rho_{0}}{6-7 \rho_{0}}\right) \approx 1.87 \cdot 10^{-27} \mathrm{~cm}^{2},
$$

where $\alpha=1.76_{7} \cdot 10^{-30} \mathrm{~m}^{3}$ (cgs units) is the molecular polarizability of nitrogen and $\rho_{0} \approx 0.01$ the depolarization [24]. Another value for the molecular polarizability of $1.74 \cdot 10^{-30} \mathrm{~m}^{3}$ (cgs) from Lide[25] yields a similar value of $1.81 \cdot 10^{-27} \mathrm{~cm}^{2}$ for the cross section. In addition, more recent studies from NAus et al.[26] yield $1.87 \cdot 10^{-27} \mathrm{~cm}^{2}$ (theoretical) and $1.87 \cdot 10^{-27} \mathrm{~cm}^{2}$ (experimental). Therefore, $(1.84 \pm 0.03) \cdot 10^{-27} \mathrm{~cm}^{2}$ is taken as mean value for the RAYLEIGH cross section.

Although the calculation of the RAYLEIGH cross section is very simple, one has to take care because of the difference between the integral cross section and the differential cross section $\mathrm{d} \sigma / \mathrm{d} \Omega$. For the LIF case the latter is just $\sigma_{\mathrm{LIF}} / 4 \pi$ because of the isotropic fluorescence due to the fast rotation of the molecules. This is different for the RAYLEIGH scattered light, because it is scattered immediately and the polarization of the laser determines also the polarization of the scatterer. As a result, the differential cross section in the direction of the laser polarisation is very small and the cross section perpendicular to the laser polarization larger than just $\sigma_{\mathrm{RAY}} / 4 \pi$, namely $3 / 2 \sigma_{\mathrm{RAY}} / 4 \pi[24,23]$. Therefore, the polarization of the laser light is important, which should be perpendicular to the observation direction as in this experiment. As a result, the anisotropic cross section of the Rayleigh scattering yields mainly a factor of $3 / 2$ in comparison to the isotropic cross section for the LIF signal.

The last factor of equation (10) is the ratio of the density of the RAYLEIGH scatterer $n_{\text {RAY }}$ to the RAYLEIGH scattering signal represented by the original signal strength $\hat{y}_{\text {RAY }}$. The scattering experiment is performed in pure nitrogen without plasma, so that the scatterer is the ground state of the nitrogen molecule. Unfortunately, one cannot measure the RAYLEIGH scattered light without the reflections of the laser at the walls of the vacuum chamber and the electrodes. Therefore, the pressure was varied between an empty chamber and atmospheric pressure, and the measured signal is plotted in figure 6. As expected, the measurement points are on a straight line with an intercept, which represents the pressure independent signal due to reflections at the wall. The slope gives the (inverted) ratio $n_{\mathrm{RAY}} / \hat{y}_{\mathrm{RAY}}=(2.95 \pm 0.07) \cdot 10^{22} \mathrm{~cm}^{-3}$ of the scatter density to the measured voltage.

Altogether, using equation (10) for the example of $200 \mathrm{~Pa}$ and $10 \mathrm{~W}$ (figure 3) a density of $(3.4 \pm 0.6) \cdot 10^{12} \mathrm{~cm}^{-3}$ can be calculated for a distance of $6 \mathrm{~mm}$ from the powered electrode. 


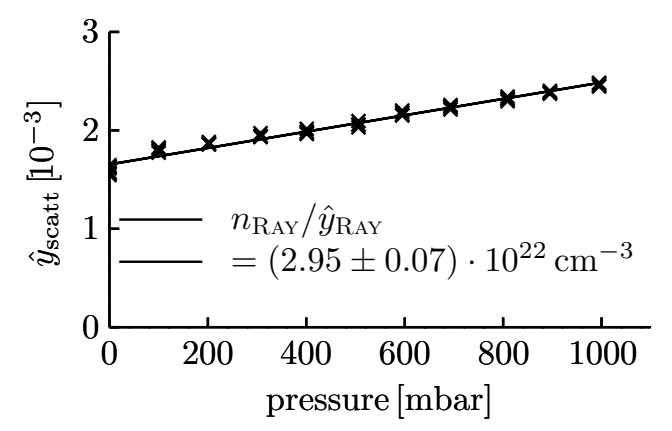

Figure 6. Pressure dependent scattering signal. The slope represents the ratio of the signal due to RAYLEIGH scattering and the (ground state) density of nitrogen.

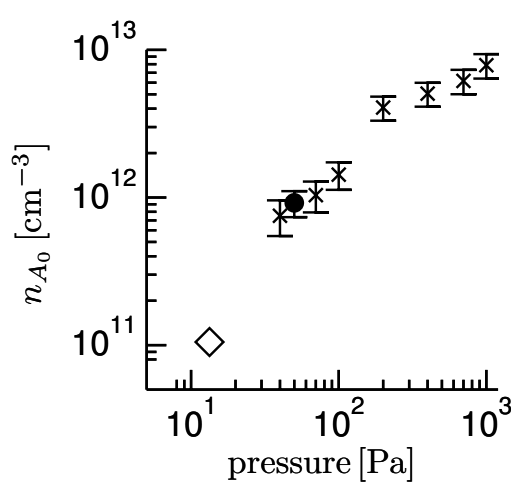

Figure 7. Maximal $A^{3} \Sigma_{u}^{+}, v=0$ densities for pressures from 40 to $1000 \mathrm{~Pa}$ and comparison with literature $(\diamond[4], \bigcirc[5])$.

\subsection{Absolute densities and axial profiles}

In this section, the measured $A^{3} \Sigma_{u}^{+}, v=0$ densities, their parameter dependencies and axial density profiles are discussed. First of all, the pressure dependence of the maximum metastable density is shown in figure 7 .

As it is expected, the metastable densities increase with pressure. This increase is steeper for low pressures and flatter for larger pressure. Furthermore, a jump is observed between 100 and $200 \mathrm{~Pa}$, which might be just due to the different rf power of these measurements (see discussion below). The agreement with the literature is good, although the small discharge gap of $10 \mathrm{~mm}$ in this experiment differs strongly from the open discharge configuration with one planar electrode in [5]. Therefore, the densities in [5] should be larger due to reduced losses at the electrodes, but this might have been compensated by the missing factor of $3 / 2$ from the comparison of the angular dependent RAYLEIGH cross section to the LIF cross section in equation (10).

Axial density profiles of $A^{3} \Sigma_{u}, v=0$ for various pressures are plotted in figure 8 . In addition, the plasma induced optical emission of the transition from $B^{3} \Pi_{g}, v=3$ to $A^{3} \Sigma_{u}^{+}, v=1$ is shown for comparison. The measurements were done for the lowest possible rf power to avoid heating of the background gas which makes the analysis more 


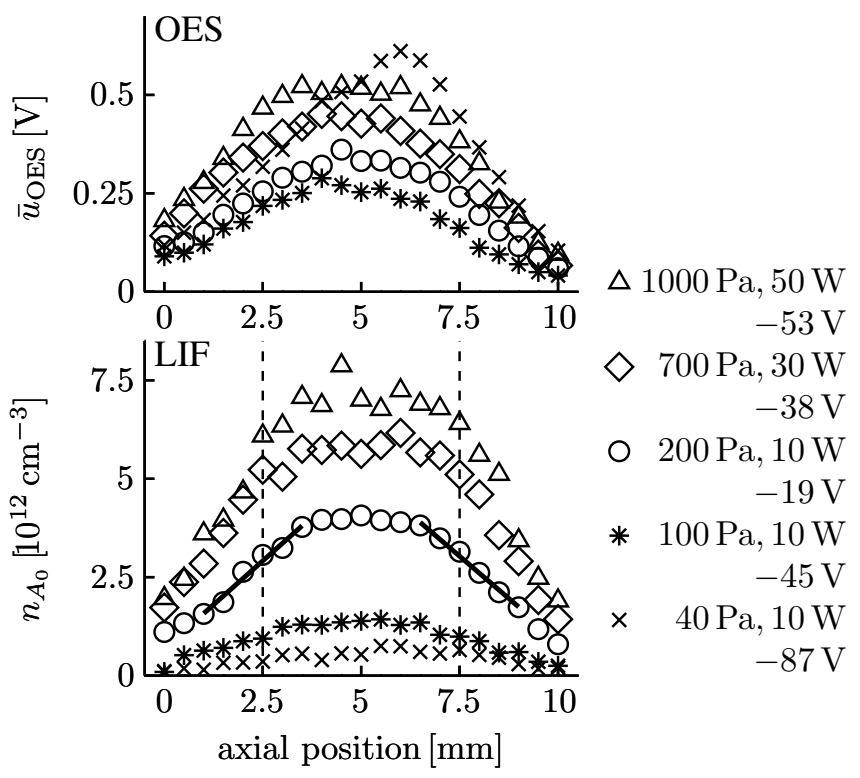

Figure 8. Axial optical emission profiles of $B^{3} \Pi_{g}, v=3 \rightarrow A^{3} \Sigma_{u}^{+}, v=1$ and density profiles of $A^{3} \Sigma_{u}^{+}, v=0$ for various pressures. The powered electrode is at $0 \mathrm{~mm}$.

difficult. The profiles are almost symmetric because of the small self-bias voltage at the powered electrode, which causes a nearly symmetric profile of the time averaged electric potential. Only for $40 \mathrm{~Pa}$ the asymmetric character is clearly visible for the plasma induced optical emission. The reason is the large sheath width at $40 \mathrm{~Pa}$ in front of the powered electrode, which is even larger than half of the discharge gap. For larger pressure, at 700 and $1000 \mathrm{~Pa}$, the optical emission at $2.5 \mathrm{~mm}$ is slightly higher than at $7.5 \mathrm{~mm}$ which reflects the asymmetry of the discharge. For the density profiles of $A^{3} \Sigma_{u}^{+}$, such an effect is not visible and the density is nearly constant in the middle of the discharge due to the large effective lifetime of this state. Only nearby the metallic electrodes, the de-excitation processes of $A^{3} \Sigma_{u}^{+}$cause a steep decrease of the density.

To compare the losses in the volume with the losses due to diffusion one can consider approximately a cylindrical volume in the discharge centre with the height of $5 \mathrm{~mm}$ corresponding to the difference between the axial positions of 2.5 and $7.5 \mathrm{~mm}$, and the cross section area $A$. Within this region the metastable density is assumed to be constant. A comparison of the different quenching processes in the discharge centre is given in table 1. Despite the clearly larger ground state density, the quenching by the background gas is negligible and the pooling reaction dominates in pure nitrogen. Furthermore, the quenching by oxygen is also large, which will be discussed below. Besides the pooling reaction as the most important loss process in the volume, the diffusion and the loss at the electrodes is also important. Considering the assumed cylinder in the discharge middle, the diffusion in the radial direction is negligible because of the large electrode area in comparison to the small discharge gap, which implies a homogeneous discharge in the radial direction nearby the discharge axis. The axial 
Table 1. Densities of important quenching partners and the corresponding quenching rates at $300 \mathrm{~K}$ for 40,200 and $1000 \mathrm{~Pa}$.

\begin{tabular}{|c|c|c|c|c|c|c|}
\hline & & ref. & $40 \mathrm{~Pa}$ & $200 \mathrm{~Pa}$ & $1000 \mathrm{~Pa}$ & \\
\hline density of ground state $X^{1} \Sigma_{g}^{+}$ & $n_{X}$ & & 9.7 & 48 & 240 & $10^{15} \mathrm{~cm}^{-3}$ \\
\hline density of $A^{3} \Sigma_{u}^{+}, v=0$ in discharge centre & $n_{A_{0}}$ & & $\sim 0.6$ & $\sim 4.0$ & $\sim 7.1$ & $10^{12} \mathrm{~cm}^{-3}$ \\
\hline density of $\mathrm{O}_{2}$ for $1 \%$ oxygen admixture & $n_{\mathrm{O}_{2}}$ & & & 5 & & $10^{14} \mathrm{~cm}^{-3}$ \\
\hline $\mathrm{N}_{2}\left(A^{3} \Sigma_{u}^{+}\right)+\mathrm{N}_{2}\left(X^{1} \Sigma_{g}^{+}\right) \rightarrow$ products & $n_{A_{0}} n_{X} \cdot 3.0 \cdot 10^{-16} \mathrm{~cm}^{3} \mathrm{~s}^{-1}$ & {$[28]$} & 1.7 & 58 & 510 & $10^{12} \mathrm{~cm}^{-3} \mathrm{~s}^{-1}$ \\
\hline $\mathrm{N}_{2}\left(A^{3} \Sigma_{u}^{+}\right)+\mathrm{N}_{2}\left(A^{3} \Sigma_{u}^{+}\right) \rightarrow$ products & $n_{A_{0}}^{2} \cdot 4 \cdot 10^{-10} \mathrm{~cm}^{3} \mathrm{~s}^{-1}$ & {$[29]$} & 1.4 & 64 & 200 & $10^{14} \mathrm{~cm}^{-3} \mathrm{~s}^{-1}$ \\
\hline & $n_{A_{0}}^{2} \cdot 1.5 \cdot 10^{-10} \mathrm{~cm}^{3} \mathrm{~s}^{-1}$ & {$[30]$} & 0.5 & 24 & 76 & $10^{14} \mathrm{~cm}^{-3} \mathrm{~s}^{-1}$ \\
\hline $\mathrm{N}_{2}\left(A^{3} \Sigma_{u}^{+}\right)+\mathrm{O}_{2} \rightarrow$ products & $n_{A_{0}} n_{\mathrm{O}_{2}} \cdot 2.5 \cdot 10^{-12} \mathrm{~cm}^{3} \mathrm{~s}^{-1}$ & {$[29]$} & & 50 & & $10^{14} \mathrm{~cm}^{-3} \mathrm{~s}^{-1}$ \\
\hline
\end{tabular}

Table 2. Quantification of the diffusion losses at discharge centre for 40, 200 and $1000 \mathrm{~Pa}$.

\begin{tabular}{lrrrr} 
& $40 \mathrm{~Pa}$ & $200 \mathrm{~Pa}$ & $1000 \mathrm{~Pa}$ \\
\hline mean axial density gradient $\mathrm{d} n_{A_{0}} / \mathrm{d} z$ at 2.5 and $7.5 \mathrm{~mm}$ & 1.3 & 8.7 & 14 & $10^{12} \mathrm{~cm}^{-4}$ \\
diffusion coefficient $D$ for $\mathrm{N}_{2}\left(A^{3} \Sigma_{u}^{+}\right)$in $\mathrm{N}_{2}$ & 525 & 105 & 21 & $\mathrm{~cm}^{2} \mathrm{~s}^{-1}$ \\
diffusion current density $j=D \mathrm{~d} n_{A_{0}} / \mathrm{d} z$ & 6.8 & 9.1 & 2.9 & $10^{14} \mathrm{~cm}^{-2} \mathrm{~s}^{-1}$ \\
loss rate in discharge centre by diffusion $2 j / 0.5 \mathrm{~cm}$ & 27 & 37 & 12 & $10^{14} \mathrm{~cm}^{-3} \mathrm{~s}^{-1}$
\end{tabular}

diffusion losses out of the cylinder can be estimated by the density gradients at $2.5 \mathrm{~mm}$ and $7.5 \mathrm{~mm}$. These gradients are determined by a linear fit in figure 8 (exemplary for $200 \mathrm{~Pa}$ ) and are noted in table 2 . In addition, the diffusion coefficient $D$ for the excited $\mathrm{N}_{2}\left(A^{3} \Sigma_{u}^{+}\right)$molecule in $\mathrm{N}_{2}$ is given from $D p=2.1 \cdot 10^{4} \mathrm{~Pa} \cdot \mathrm{cm}^{2} \mathrm{~s}^{-1}[27]$. The resulting diffusion current density $j=D \cdot \mathrm{d} n_{A_{0}} / \mathrm{d} z$ is nearly equal for 40 and $200 \mathrm{~Pa}$ due to the $1: 1$ increase of the metastable density with pressure (see figure 7 ). Between 200 and $1000 \mathrm{~Pa}$, the metastable density only doubles. Hence, the density gradient is only slightly larger and the diffusion current decreases clearly due to the five times smaller diffusion coefficient. To estimate the diffusion losses in the cylinder, the diffusion current must be multiplied by the cylinder cross section area $A$ and a factor 2 for the both sides. To get the losses per volume, this quantity is divided by the cylinder volume $A \cdot 5 \mathrm{~mm}$, which cancels out the cylinder cross section area.

The comparison of the pooling reaction as the most important volume process with the diffusion shows, that for $40 \mathrm{~Pa}$ the diffusion is most important, whereas for $1000 \mathrm{~Pa}$ the pooling reaction dominates. A transition region exists at $200 \mathrm{~Pa}$, where both processes have the same order of magnitude.

Due to the different loss processes one would expect different shapes of the density profiles. Therefore, the normalized densities are plotted in figure 9. Nevertheless, the shapes of the density profiles are the same. Only for $40 \mathrm{~Pa}$ some values differ due to the larger error for these measurements.

Another important discharge parameter is the rf power respectively the resulting self-bias voltage which is more characteristic for the rf plasma. Therefore, the optical emission from $B^{3} \Pi_{g}, v=3$ to $A^{3} \Sigma_{u}^{+}, v=1$ and the densities of $A^{3} \Sigma_{u}^{+}, v=0$ are plotted again for different rf power in figure 10. Firstly, as it is expected, the asymmetric character of the optical emission profiles increases with the self-bias voltage and the maximum shifts toward the powered electrode, because electron impact excitation is the most important excitation process, located at the sheath edge [6]. In contrast 


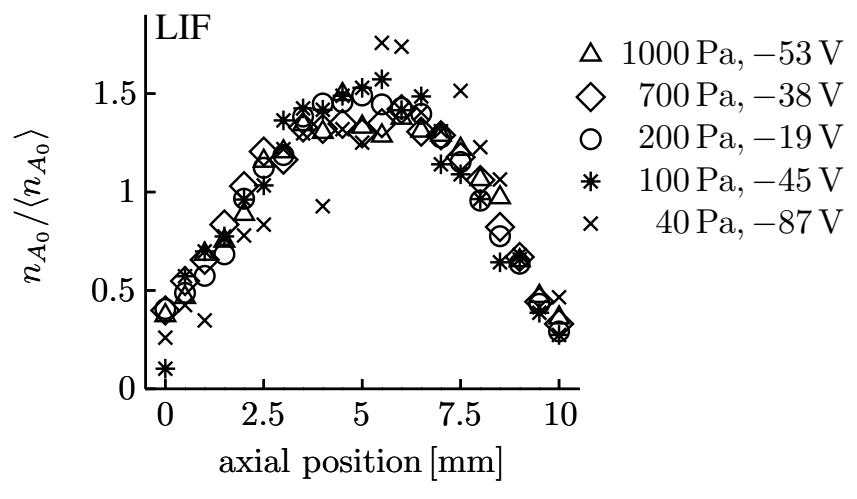

Figure 9. Normalized axial density profiles of $A^{3} \Sigma_{u}^{+}, v=0$ for various pressures. The powered electrode is at $0 \mathrm{~mm}$.

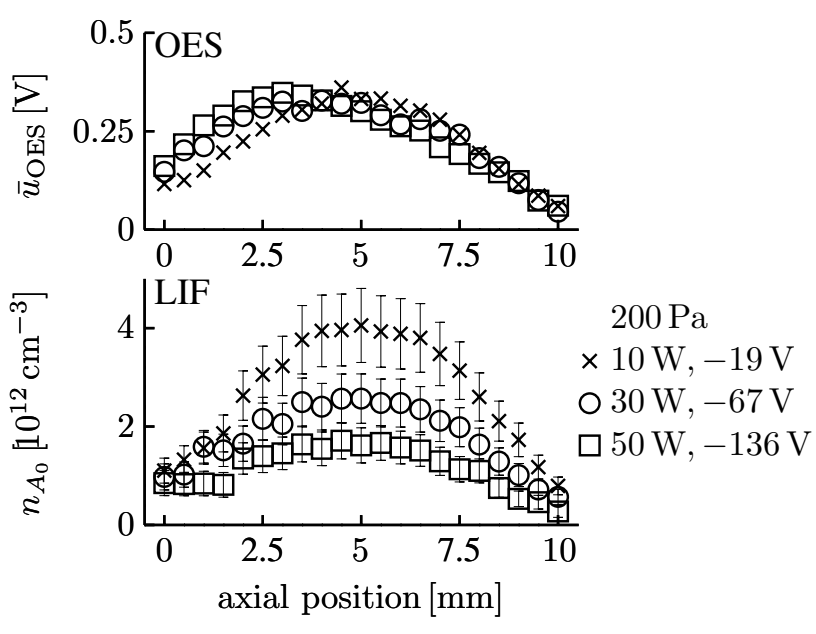

Figure 10. Axial optical emission profiles of $B^{3} \Pi_{g}, v=3 \rightarrow A^{3} \Sigma_{u}^{+}, v=1$ and density profiles of $A^{3} \Sigma_{u}^{+}, v=0$ for $200 \mathrm{~Pa}$ for various rf powers. The powered electrode is at $0 \mathrm{~mm}$.

to the optical emission, the $A^{3} \Sigma_{u}^{+}, v=0$ density profiles are still symmetric, whereas the absolute $A^{3} \Sigma_{u}^{+}, v=0$ density decreases with increasing self-bias voltage. This is a big difference to the measurements at lower pressures (and larger gaps) in [6] in a capacitively coupled rf discharge and [8] in an inductively coupled rf discharge, where the density increases with the self-bias voltage resp. rf power. One reason is the decrease of the LIF cross section due to the change in the rotational distribution caused by the temperature increase with rf power (cp. figure 5), which is not considered in the calibration due to the unknown absolute temperature. But this cannot explain the large decrease in figure 10. Reconsidering the results in [6], the increase is also related to an increase of the $B^{3} \Pi_{g}$ density. This is not the case in our measurements, because the mean optical emission from $B^{3} \Pi_{g}, v=3$ to $A^{3} \Sigma_{u}^{+}, v=1$ remains nearly constant. Hence, assuming that the cascade processes from the larger triplet states is most important one for the production of $A^{3} \Sigma_{u}^{+}$, this means that the production rate should also be nearly 


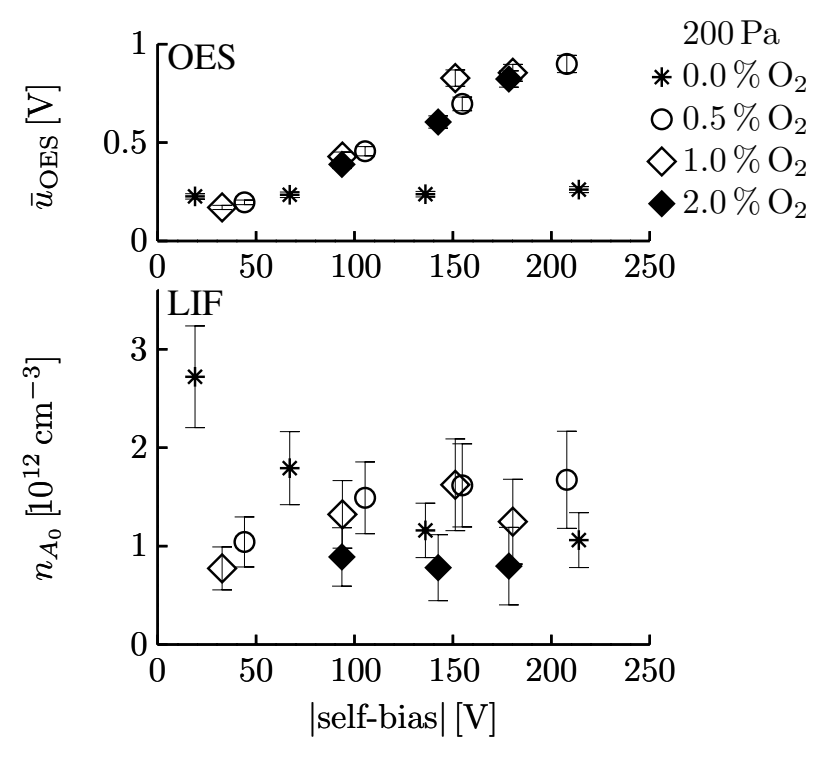

Figure 11. Mean optical emission of $B^{3} \Pi_{g}, v=3 \rightarrow A^{3} \Sigma_{u}^{+}, v=1$ and mean densities of $A^{3} \Sigma_{u}^{+}, v=0$ at $200 \mathrm{~Pa}$ depending on self-bias voltages for pure nitrogen and small oxygen admixtures.

constant with self-bias voltage. Therefore, the dependence of the $A^{3} \Sigma_{u}^{+}, v=0$ density on self-bias voltage is determined by the loss processes. These loss processes are also temperature dependent and increase with rf power. The rate coefficient of the pooling reaction is approximately proportional to $\sqrt{T}$ due to the larger thermal velocity. The other loss process, the diffusion, is determined by the diffusion coefficient $D$, which also depends on temperature. Firstly, $D$ is proportional to $1 / n$, which results for constant pressure in a linear increase with temperature. Secondly, the factor $\sqrt{T}$ results again from the thermal velocity, so altogether $D \propto T^{3 / 2}$, which means a significant increase of the diffusion coefficient with temperature. Assuming again a temperature of $350 \mathrm{~K}$ at $10 \mathrm{~W}$ and $550 \mathrm{~K}$ at $50 \mathrm{~W}$, the diffusion coefficient enlarges by a factor of 2 . In summary, the temperature increase with power explains the decrease in metastable density.

\subsection{Small oxygen admixtures}

As it is known from literature, the quenching of $A^{3} \Sigma_{u}^{+}$by oxygen is much larger than by nitrogen itself, see table 1 . For an admixture of $1 \%$ oxygen the loss rate has the same order of magnitude as the pooling reaction in the discharge center. At the boundaries, it even exceeds the pooling reaction and becomes the dominant loss process. Furthermore, for large dissociation degrees of oxygen the losses by quenching due to atomic oxygen become dominante, because of the large quenching rate of $2.7 \cdot 10^{-11} \mathrm{~cm}^{3} \mathrm{~s}^{-1}[29]$. However, the admixture of oxygen should decrease the $A^{3} \Sigma_{u}^{+}$ density significantly. Surprisingly, this is not always the case, as it is seen from figure 11, where the mean plasma induced optical emission and the $A^{3} \Sigma_{u}^{+}$densities are plotted versus the self-bias voltage for various oxygen admixtures. 
In the case of pure nitrogen plasma, the density of $A^{3} \Sigma_{u}^{+}$decreases with rf power as it is discussed above. From 20 to $220 \mathrm{~V}$, a factor of 2.5 is visible. In addition, the related optical emission intensity remains constant. In contrast to that, in the case of an oxygen admixture of $0.5 \%$ the $A^{3} \Sigma_{u}^{+}$density starts at a lower level and increases to a maximum with a larger $A^{3} \Sigma_{u}^{+}$density compared with pure nitrogen. The 2.5 times lower density at small rf power is due to the larger quenching mentioned above. The increase for larger self-bias voltage is explainable by the increasing optical emission, which is a measure for the production rate due to the cascade process $C^{3} \Pi_{u} \rightarrow B^{3} \Pi_{g} \rightarrow A^{3} \Sigma_{u}^{+}$. From $50 \mathrm{~V}$ selfbias to $210 \mathrm{~V}$ it increases by a factor of 5 with $0.5 \%$ oxygen. Comparing the metastable densities for pure nitrogen and $0.5 \%$ oxygen at $210 / 220 \mathrm{~V}$, the twice larger value for the case with oxygen inside is explainable by the 2.5 times larger quenching and the 5 times larger production from $B^{3} \Pi_{g}$. For larger oxygen concentrations, the optical emission is similar to the case of $0.5 \%$ oxygen admixture, so that the larger quenching rates result in a lower $A^{3} \Sigma_{u}^{+}$density as expected.

\section{Conclusions}

The density of the lowest metastable $A^{3} \Sigma_{u}^{+}$state of nitrogen in its vibrational ground state was successfully measured by laser induced fluorescence spectroscopy in combination with an absolute density calibration by RAYLEIGH scattering. The different time response of both signals allowed to measure the effective lifetime of the excited $B^{3} \Pi_{g}$ state and to calculate densities without the large uncertainties of the quenching coefficients, even for pressures up to $1000 \mathrm{~Pa}$.

The axial density profiles for the low pressure capacitively coupled radio-frequency discharges show similar shapes, although the dominating loss process for low pressures is diffusion to the electrodes and for larger pressure the collisional quenching within the volume. The increase of discharge power resp. self-bias voltage results in a lower metastable density, which can be explained by an increase of the rotational and kinetic (gas) temperature, respectively. Furthermore, the admixture of oxygen causes the well known decrease of the density due to the larger quenching, on the other hand it increases also the production of $A^{3} \Sigma_{u}^{+}$due to the cascade processes from the higher triplet states so that the $A^{3} \Sigma_{u}^{+}$density exceeds the value for the pure nitrogen case for some parameter sets.

[1] F. R. Gilmore, R. R. Laher, and P. J. Espy. Franck-Condon factors, $r$-centroids, electronic transition moments, and Einstein coefficients for many nitrogen and oxygen band systems. $J$. Phys. Chem. Ref. Data, 21:1005-1107, 1992.

[2] A. Lofthus and P. H. Krupenie. The spectrum of molecular nitrogen. J. Phys. Chem. Ref. Data, 6:113-307, 1977.

[3] S. De Benedictis, G. Dilecce, and M. Simek. Time-resolved LIF spectroscopy on $\mathrm{N}_{2}(\mathrm{~A})$ metastable in a $\mathrm{He} / \mathrm{N}_{2}$ pulsed rf discharge. Chemical Physics, 178(1-3):547 - 560, 1993.

[4] S. De Benedictis, G. Dilecce, and M. Simek. LIF measurement of $\mathrm{N}_{2}\left(A^{3} \Sigma_{u}^{+}, v=4\right)$ population density in a pulsed rf discharge. J. Phys. D: Appl. Phys., 31:1197-1205, 1998.

[5] B. Krames, Th. Glenewinkel-Meyer, and J. Meichsner. In situ determination of absolute number 
densities of nitrogen molecule triplet states in an rf-plasma sheath. J. Appl. Phys., 89(6):31153120, 2001.

[6] B. Krames, T. Glenewinkel-Meyer, and J. Meichsner. Spatial density profiles of the $\mathrm{N}_{2}$ metastable state $A^{3} \Sigma_{u}^{+}$, the $B^{3} \Pi_{g}$ and $C^{3} \Pi_{u}$ states in an asymmetric rf discharge. J. Phys. D: Appl. Phys., 34:1789-1798, 2001.

[7] Satoshi Matsuda, Hirotsugu Shimosato, Motoshige Yumoto, and Takao Sakai. Detection of nitrogen metastable molecules by using the threshold ionization mass spectrometry. Electrical Engineering in Japan, 147(1):17-24, 2004.

[8] G. Hancock, R. Peverall, G. A. Ritchie, and L. J. Thornton. Absolute number densities of vibrationally excited $\mathrm{N}_{2}\left(A^{3} \Sigma_{u}^{+}\right)$produced in a low pressure rf plasma. J. Phys. D: Appl. Phys., 39:1846-1852, 2006.

[9] Yoshimine Horikawa, Kazuaki Kurihara, and Koichi Sasaki. Absolute densities of $\mathrm{N}_{2}\left(A^{3} \Sigma_{\mathrm{u}}^{+}\right)$, $\mathrm{N}\left({ }^{4} S^{\circ}\right)$, and $\mathrm{N}\left({ }^{2} D^{\circ}\right)$ in an inductively coupled nitrogen plasma source. Japanese Journal of Applied Physics, 49(2):026101, 2010.

[10] Gabi D. Stancu, Mario Janda, Farah Kaddouri, Deanna A. Lacoste, and Christophe O. Laux. Time-resolved CRDS measurements of the $\mathrm{N}_{2}\left(A^{3} \Sigma_{u}^{+}\right)$density produced by nanosecond discharges in atmospheric pressure nitrogen and air. The Journal of Physical Chemistry A, 114(1):201-208, 2010.

[11] Giorgio Dilecce, Paolo Francesco Ambrico, and Santolo De Benedictis. Optical-optical double resonance LIF detection of $\mathrm{N}_{2}\left(A^{3} \Sigma_{u}^{+}\right)$in high pressure gas discharges. Plasma Sources Science and Technology, 14(3):561, 2005.

[12] G. Dilecce, P. F. Ambrico, and S. De Benedictis. $\mathrm{N}_{2}\left(A^{3} \Sigma_{u}^{+}\right)$density measurement in a dielectric barrier discharge in $\mathrm{N}_{2}$ and $\mathrm{N}_{2}$ with small $\mathrm{O}_{2}$ admixtures. Plasma Sources Sci. Technol., 16:511$522,2007$.

[13] P. F. Ambrico, M. Simek, G. Dilecce, and S. De Benedictis. On the measurement of $\mathrm{N}_{2}\left(A^{3} \Sigma_{u}^{+}\right)$ metastable in $\mathrm{N}_{2}$ surface-dielectric barrier discharge at atmospheric pressure. Plasma Chem. Plasma Process., 28(3):299-316, 2008.

[14] M. Simek, P. F. Ambrico, S. De Benedictis, G. Dilecce, V. Prukner, and J. Schmidt. $\mathrm{N}_{2}\left(A^{3} \Sigma_{u}^{+}\right)$ behaviour in a $\mathrm{N}_{2}-\mathrm{NO}$ surface dielectric barrier discharge in the modulated ac regime at atmospheric pressure. J. Phys. D: Appl. Phys., 43:124003, 2010.

[15] R. Ono, C. Tobaru, Y. Teramoto, and T. Oda. Laser-induced fluorescence of $\mathrm{N}_{2}\left(A^{3} \Sigma_{u}^{+}\right)$metastable in $\mathrm{N}_{2}$ pulsed positive corona discharge. Plasma Sources Sci. Technol., 18:025006, 2009.

[16] Yoshiyuki Teramoto, Ryo Ono, and Tetsuji Oda. Measurement of metastable in $\mathrm{N}_{2}$ pulsed positive corona discharge with trace amounts of additives. Journal of Physics D: Applied Physics, 42(23):235205, 2009.

[17] C. O. Laux and C. H. Kruger. Arrays of radiative transition probabilities for the $\mathrm{N}_{2}$ first and second positive, no beta and gamma, $\mathrm{N}_{2}^{+}$first negative, and $\mathrm{O}_{2}$ Schuhmann-Runge band systems. J. Quant. Spectrosc. Radiat. Transfer, 48(1):9-24, 1992.

[18] L. G. Piper. State-to-state $\mathrm{N}_{2}\left(A^{3} \Sigma_{u}^{+}\right)$energy pooling reactions. II. The formation and quenching of $\mathrm{N}_{2}\left(B^{3} \Pi_{g}, v^{\prime}=1-12\right)$. J. Chem. Phys., 88(11):6911-6921, 1988.

[19] S. De Benedictis, G. Dilecce, and M. Simek. Excitation and decay of $\mathrm{N}_{2}\left(B^{3} \Pi_{g}, v\right)$ states in a pulsed discharge: Kinetics of electrons and long-lived species. J. Chem. Phys., 110:2947-2962, 1999.

[20] A. Rotem, I. Nadler, and S. Rosenwaks. Laser-induced fluorescence studies of collisional coupling of $\mathrm{N}_{2}\left(B^{3} \Pi_{g}\right)$ with $\mathrm{N}_{2}\left(W^{3} \Delta_{u}\right)$ and $\mathrm{N}_{2}\left(A^{3} \Sigma_{u}^{+}\right)$. Chem. Phys. Letters, 83(2):281-286, 1981.

[21] N. Sadeghi and D. W. Setser. Collisional coupling and relaxation of $\mathrm{N}_{2}\left(B^{3} \Pi_{g}\right)$ and $\mathrm{N}_{2}\left(W^{3} \Delta_{u}\right)$ vibrational levels in Ar and Ne. J. Chem. Phys., 79:2710-2726, 1983.

[22] F. Roux, F. Michaud, and J. Verges. High-resolution Fourier spectrometry of ${ }^{14} \mathrm{~N}_{2}$ infrared emission spectrum: Extensive analysis of the $B^{3} \Pi_{g}-A^{3} \Sigma_{u}^{+}$system. J. Mol. Spectrosc., 97:253265, 1983.

[23] R. B. Miles, W. R. Lempert, and J. N. Forkey. Laser Rayleigh scattering. Meas. Sci. Technol., 
12:R33-R51, 2001.

[24] N. J. Bridge and A. D. Buckingham. The polarisation of laser light scattered gases. Proc. Roy. Soc. A, 295:334-349, 1966.

[25] D. R. Lide. CRC Handbook of Chemistry and Physics. CRC Press LCC, 2001.

[26] H. Naus and W. Ubachs. Experimental verification of Rayleigh scattering cross sections. Optics Letters, 25(5):347-349, 2000.

[27] I. M. Stewart. The reflection of metastable particles at a surface. Journal of Physics D: Applied Physics, 27(7):1487, 1994.

[28] G. Cartry, L. Magne, and G. Cernogora. Experimental study and modelling of a low-pressure $\mathrm{N}_{2}-\mathrm{O}_{2}$ time afterglow. J. Phys. D: Appl. Phys., 32:1894-1907, 1999.

[29] J. T. Herron. Evaluated chemical kinetics data for reactions of $\mathrm{N}\left({ }^{2} D\right), \mathrm{N}\left({ }^{2} P\right)$, and $\mathrm{N}_{2}\left(A^{3} \Sigma_{u}^{+}\right)$in the gas phase. J. Chem. Phys. Ref. Data, 28(5):1453-1483, 1999.

[30] L. G. Piper. State-to-state $\mathrm{N}_{2}\left(A^{3} \Sigma_{u}^{+}\right)$energy-pooling reactions. I. The formation of $\mathrm{N}_{2}\left(C^{3} \Pi_{u}\right)$ and the Herman infrared systems. J. Chem. Phys., 88:231-239, 1988. 